\title{
Lassa fever Outbreak in Makurdi, North Central Nigeria: What You Need To Know
}

\author{
${ }^{1}$ Godwin I. Achinge, ${ }^{2}$ Jonathan. T. Kur, ${ }^{3}$ Shima K. Gyoh \\ ${ }^{l}$ Department of Medicine, Benue State University Teaching Hospital, Makurdi, Nigeria \\ ${ }^{2}$ Epidemiological Unit, Benue State Ministry of Health, Makurdi, Nigeria \\ ${ }^{3}$ Department of Surgery, Benue State University Teaching Hospital, Makurdi, Nigeria
}

\begin{abstract}
Introduction: The Nigerian Federal Ministry of health reports indicate that Lassa fever outbreaks occur very frequently in different parts of Nigeria. Most of the cases pass unnoticed because the index of suspicion has remained low. The recent Makurdi outbreak was unnoticed for several weeks and killed several people including a Physician before detection. This review aims to remind health workers and enlighten the general public on the danger of Lassa fever.

Methods: The Makurdi epidemic was used as a case study to review Lassa fever in Nigeria. Data from the Federal Ministry of Health, the World Health Organisation and published articles on Lassa fever were reviewed.We acknowledge the Federal Ministry of Health Outbreak Investigation and Control Team that worked with us in Benue during the outbreak for sharing the Nigerian Lassa fever data with us. We equally are grateful to the Disease Prevention and Control (DPC) Unit, World Health Organization Country Office, Abuja, Nigeria for providing resource materials on prevention of Lassa fever.
\end{abstract}

Conclusion: A high index of suspicion is crucial to early detection and treatment of Lassa fever and strict adherence to universal precautions would prevent nosocomial transmission.

Key Words: Lassa fever, outbreak, Makurdi

\section{Introduction}

In January 2013, there was an outbreak of Lassa fever in Makurdi, the capital of Benue State in north central Nigeria which claimed several lives. The diagnosis of this deadly zoonotic haemorrhagic fever at the Benue State University Teaching Hospital (BSUTH), Makurdi, created panic among inhabitants of Makurdi town in particular and Benue state in general. Since the beginning of the outbreak, there were many stories and rumours on what Lassa fever is, how it comes about, its clinical presentation, how to deal with a suspected case of Lassa fever and how to prevent it.

Reports from the Nigerian Federal Ministry of health indicate that Lassa fever outbreaks occur very frequently in different parts of Nigeria ${ }^{1-3}$ However, most of the infections pass unnoticed because the clinical presentation may be indistinguishable from other fevers like malaria or typhoid. ${ }^{4}$ The index of suspicion for this deadly infectious medical condition has remained very low, therefore, physicians often miss the diagnosis even when the patients present with classical features of Lassa fever. ${ }^{5}$ On many occasions, patients infected with Lassa fever are admitted into the wards, given healthcare for several days and sometime die without a suspicion of the underlying danger. These patients are nursed together with other patients often without necessary precaution. The missed diagnosis increases the case fatality rate among those infected with Lassa fever as well as the chances of transmitting infection to close contacts and healthcare workers. ${ }^{5}$

Lassa fever outbreaks often affect healthcare providers sometimes with fatal consequences. ${ }^{4-7}$ The latest Makurdi outbreak killed a Physician who had earlier treated a patient with symptoms consistent with Lassa fever without suspecting Lassa fever. The Physician referred the patient to the Benue State University Teaching Hospital, Makurdi where the patient died a few hours on arrival at the emergency of the teaching hospital. Again, the diagnosis was missed. It was only after the Physician himself was referred to the same teaching hospital that the diagnosis of Lassa fever was made and contact tracing revealed the index case and others.

This review aims to remind health workers and enlighten the general public on the danger of Lassa fever. It highlights basic facts on Lassa fever in Nigeria and is intended to raise awareness on this deadly viral haemorrhagic fever, address some of the frequently asked questions and offer few tips on preventing further outbreaks. The review is for the general public and does not provide details on the treatment of Lassa fever.

\section{What Is Lassa fever?}

Lassa fever or Lassa hemorrhagic fever (LHF) is an acute Viral Haemorrhagic Fever (VHF) caused by the Lassa virus. The illness was discovered in 1969 when two missionary nurses died in a remote village called Lassa in Borno State, north eastern Nigeria. The cause of the illness was found to be the Lassa virus, named after the village where the fever was first reported. ${ }^{6}$ Lassa virus is a zoonotic virus that spreads to man from the 
multi-mammate rat (Mastomys natalensis) which is a common rodent in equatorial Africa, ubiquitous in human households and eaten as a delicacy in some areas. ${ }^{4}$ it is worthy of note that the Benue trough is populated by these rodents and the inhabitants of the area consider rat meat a delicacy.

\subsection{Transmission of Lassa virus}

Transmission of Lassa virus to humans occur when humans have contact with the natural hosts for the virus, the multi-mammate rats (Mastomys natalensis) by touching objects or eating food contaminated by urine or faeces of these rodents, or through cuts or sores. ${ }^{8}$ Since these rats often live in and around homes and scavenge on human food remains or poorly stored food, transmission of this sort is common. Secondly, Contact with the virus may occur when a person inhales tiny particles in the air contaminated with rodent excretions. This is called aerosol or airborne transmission. Finally, because Mastomys rodents are sometimes consumed as food in some areas, infection may occur via direct contact when they are caught and prepared for food. Remember that over $90 \%$ of the natives in the Tiv speaking areas of Benue, Nassarawa and Taraba states consider rats a delicacy. Lassa fever may also spread through person-to-person contact. This type of transmission occurs when a person comes into contact with the virus in the blood, urine, faeces, semen or other fluids of an individual infected with the Lassa virus. Person-to-person transmission is common in both community and health care settings, where, along with the above-mentioned modes of transmission, the virus also may be spread in contaminated medical equipment, such as reused needles. ${ }^{5}$ The virus is excreted in urine for three to nine weeks following infection and in semen for three months. The extent of sexual transmission is unknown.

The virus cannot be spread through casual contact (including skin-to-skin contact without exchange of body fluids). Lassa fever in Nigeria has a seasonal variation with peaks in the dry season of the year. This is most pronounced from December to April when burning of bush is at its peak.

\section{Epidemiology Of Lassa Fever In Nigeria}

Lassa fever is endemic in Nigeria with the prevalence of antibodies to the virus being approximately $21 \%$ in the Nigerian population. ${ }^{9}$ It is also endemic in Liberia, Sierra-Leone and Guinea. ${ }^{4}$ Lassa fever seropositivity has also been found in the Central African Republic, Democratic Republic of the Congo, Mali, and Senegal. ${ }^{7}$ It is estimated that, the Lassa virus infects about 300,000 - 500,000 people per year with approximately 5,000 deaths each year. ${ }^{10}$ Majority of the deaths recorded in Nigeria so far have been among the youths in their primes of life, most especially health personnel and pregnant women., ${ }^{42}$

Data from the Nigerian Federal Ministry of Health, Epidemiology Division reveal that Lassa fever has been reported in more than 23 of the 36 states of Nigeria. Ebonyi, Edo, Nassarawa and Plateau States are the most frequently affected. In recent times, frequent outbreaks have also been observed in Adamawa and Taraba states. The pattern of Lassa fever outbreaks in Nigeria over the years shows a worrying trend where outbreaks are increasingly becoming more frequent with widening geographical spread.(Fig1) Historically, it started at Lassa Village near Maiduguri, Borno State (1969), that same outbreak had a nosocomial spread to Jos, Plateau State (1969-1970). Thereafter, Lassa fever outbreaks occurred in Zonkwa, Kaduna State and Onitsha, Anambra State (1974), Pankshin, Plateau State (1976), Ekpoma, Aboh Mbaise, and Aba (1989), Ekpoma (endemic for Lassa) (1989 till date), Lafia (December 1993 to February 1994) Northern part of Edo State, including Ekpoma, Igarra, and Ibilo (2001 and 2004), Ebonyi and Ogun States (2005) Edo, Plateau, Kogi, Benue, Ondo, Nasarawa, Ebonyi (2007/2008), Edo, Nasarawa, Gombe, Kaduna,Plateau, Ondo,Lagos States and FCT(2009), Kaduna, Kebbi, Plateau, Taraba, Edo, Kogi, Ondo (2010).

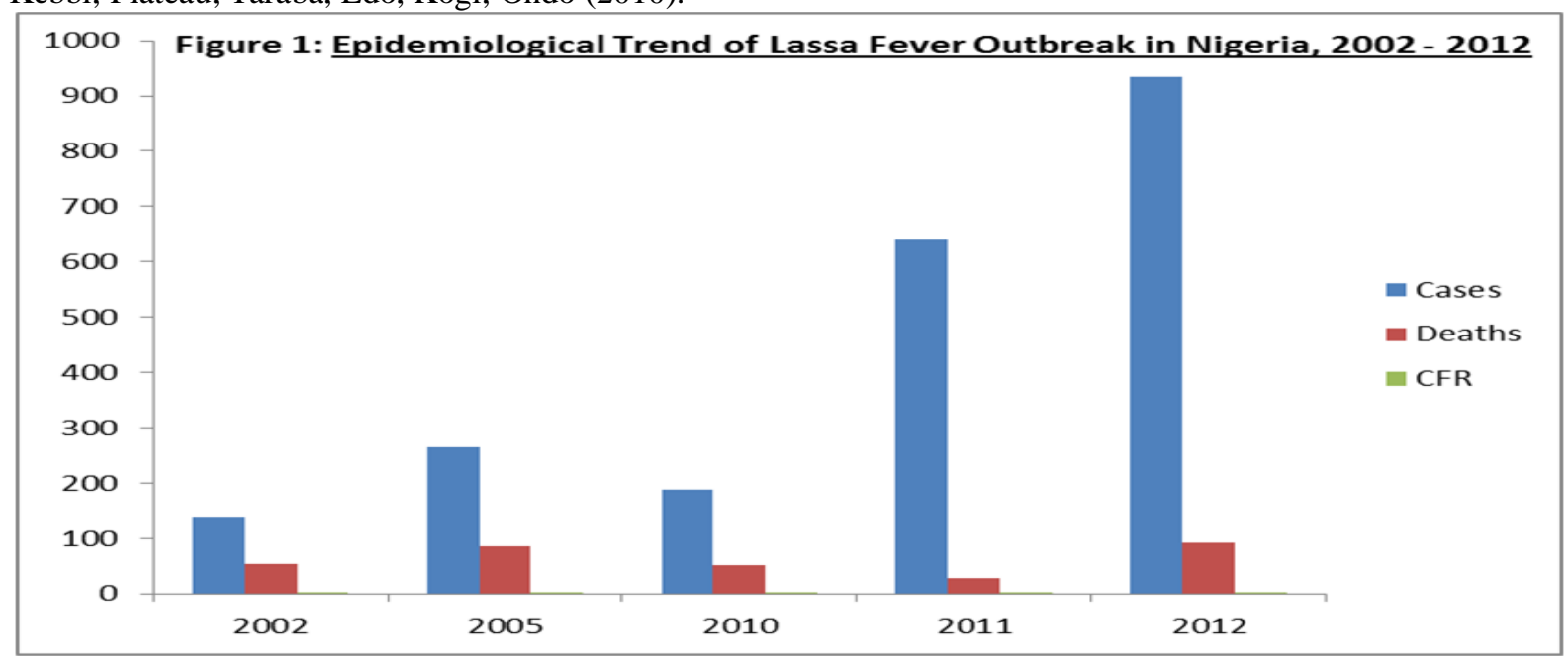


Nigeria experienced its worst outbreak of the Lassa fever December 2011 to June 2012 which peaked during the month of February 2012. (Fig 2) The outbreak involved the entire country affecting 41 LGAs in 23 States, with Edo, Taraba, Ebonyi, Rivers, Plateau and Yobe States being the most affected.

On the whole 937 cases and 95 deaths were recorded, with a Case Fatality Rate (CFR) of 10.14\%. Out of the 937 cases $148(15.7 \%)$ were laboratory confirmed. The confirmation was carried out by two laboratories located at the Lassa Research Institute, Irrua, Edo State and Lagos University Teaching Hospital, Idi-Araba Lagos.

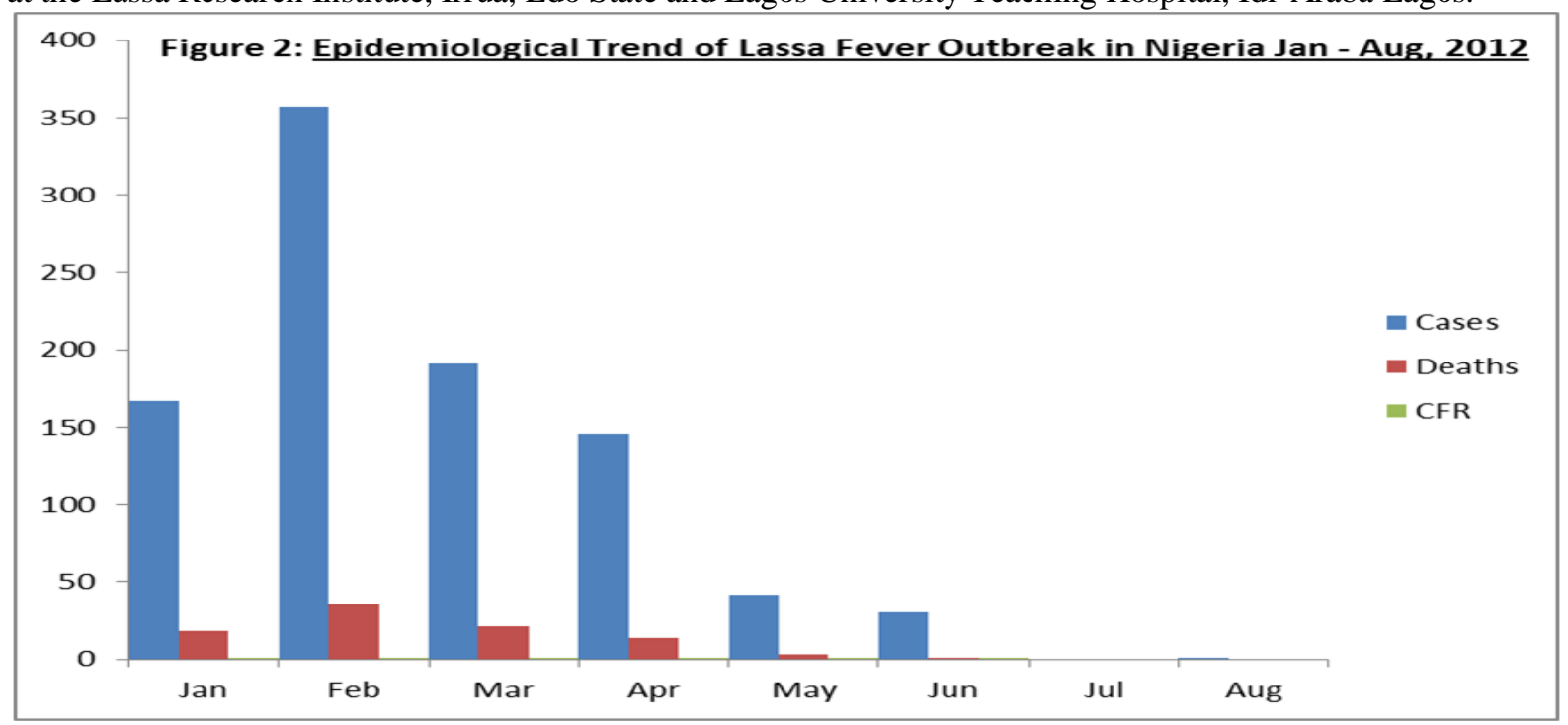

\section{Natural Course Of Lassa Fever}

While Lassa fever is mild or has no observable symptoms in about $80 \%$ of people infected with the virus, the remaining $20 \%$ have a severe multisystem disease. Approximately $15-20 \%$ of patients hospitalized for Lassa fever will die and this could cause panic with serious socio-economic consequences. The death rates are particularly high for pregnant women and their unborn babies. About one-third of those who survive may have mild to severe degrees of deafness, and in many cases the hearing loss is permanent. During epidemics, Lassa fever case-fatality rate may reach $50 \%$.

\subsection{Symptoms of Lassa fever}

It takes between 6 and 21 days for an individual to fall sick after the Lassa fever virus enters their body. Lassa fever starts with a gradual fever, a feeling of being ill and with body pain. The fever typically does not respond to anti-malaria and antibiotic treatment. After a few days, the patient may have sore throat, cough, chest and abdominal pain, headache, vomiting, diarrhoea, dizziness, red eyes and swollen face and neck. Later in the illness (after 14 days) the person may start to bleed spontaneously from the gums, in the stool, in urine, in the eyes or under the skin. The person may also have fits and die.

Although Lassa fever may start like Malaria, the fact that it does not respond to any anti-malarial drug and the typical signs of sore throat and swollen face, will warn of Lassa fever.

\subsection{Case definition for Lassa fever}

Unexplained fever $>38^{0} \mathrm{C}$ or $100.4^{0} \mathrm{~F}$ lasting for one week or more.

And one of the following: 1. No response to standard treatment for most likely cause of fever (malaria, typhoid fever, etc), 2. Readmitted within 3 weeks of inpatient care for an illness with fever

And one of the following: 1. Edema or bleeding, 2. Sore throat and retrosternal pain/vomiting, 2. Spontaneous abortion following fever, 3. Hearing loss following fever

\subsection{What should you do if you think someone has Lassa fever?}

If you have a fever, you should see your nearest health worker as soon as possible. They will probably first try to run some tests on you and treat you for malaria. If there is no recovery after 2 days or if you start to have some of the symptoms listed earlier, you should go back to the health worker. The health worker can refer you to the nearest hospital if they think that you could have Lassa fever. Pregnant women with fever and a sore throat should be transferred (referred) if there is the slightest suspicion of Lassa fever, as the disease is especially dangerous in pregnancy. There is a high likelihood that the baby may die in the womb and the woman may die after a miscarriage. 


\subsection{How is Lassa fever treated?}

Lassa fever requires specialized treatment using a drug called RIBAVIRIN. This drug is prescribed by a Specialist in the hospital where careful measures can be taken to avoid the spread of the diseases in the hospital setting. The drug is given by intravenous injections by trained health workers for a total of 10 days. Early treatment is very important for survival. The drug works best if the patient comes to the hospital within the first seven days of illness. The earlier it is started the more likely that there will be a positive outcome. Most people start to recover quickly but the sick person may continue to have Lassa fever symptoms for several days especially if the illness has been on for a long time. Although Ribavirin injection is an effective drug, it is not always certain that cure will take place in all cases as some of the affected persons will still die after they have received the drug especially if they come to the hospital late in the illness.

There is no Lassa fever vaccine at the moment to prevent the spread of Lassa fever but some research is currently being conducted in Europe and America to try and get a vaccine against the disease.

\subsection{What can I do if I have come into close contact with someone with Lassa fever?}

If you have come into close contact with someone with Lassa fever, report to the nearest health worker who will ask you some questions and examine you. The health worker will advise you to report back immediately you begin to experience any of the symptoms mentioned earlier. At this time you will be advised on any further tests that you would need.

There is currently no evidence to support the role and use of Ribavirin as post-exposure preventive treatment for Lassa fever although oral ribavirin has been given to healthcare workers and close contact during epidemics of Lassa fever.

\section{How Can You Prevent Lassa Fever?}

Lassa fever is curable but the cure is not $100 \%$ effective and so the best way is to prevent it. At the moment, there is no vaccine to prevent Lassa fever. Lassa fever can be prevented through the following: -

\subsection{Preventing the spread of the disease by rats}

1. Keep your compound clean and clear bushes around your house to prevent rats from entering your house.

2. Dispose garbage correctly and away from the house

3. Do not leave food lying on the floor because it will attract rats to your home

4. Keep food and water covered to prevent contamination by rats

5. Store your food in boxes or cabinets away from rats

6. Do not dry food materials in open places where rats can reach them

7. Kill rats using traps or rodenticides but do not touch rats with your bare hands, use a shovel, stick or plastic.

8. Bury bodies of dead rats well; do not leave the bodies of dead rats on the streets or near houses

9. Wash your hands with soap or ash if you come in contact with rats

10. Keep a cat in your house to keep the rats away. The cat cannot get Lassa fever or give you the disease

\subsection{Preventing sick people from infecting others}

1. If you take care of someone with Lassa fever at home, wash your hands with soap or ash every time you attend to them

2. If you have open cuts or wound these should be covered before you take care of a sick person

3. Avoid contact with blood, vomit, stool, urine, or semen of the person who is suffering from Lassa fever or someone who had Lassa fever in the last 2months

4. Wash all utensils, beddings and clothes of the sick or recovering person with detergent soap and bleach

5. If blood, urine, stools, or vomit of a person with Lassa fever are spilled, pour bleach on them and soak for 30mins before cleaning.

6. Do not share food, cup or spoon with someone who has got Lassa fever or who is recovering from the disease, they should use a separate bowl and cup for eating or drinking. And they should always be washed separately

\section{Acknowledgements}

We acknowledge the Federal Ministry of Health Outbreak Investigation and Control Team that worked closely with us to contain the outbreak in Benue State, for sharing Nigerian Lassa fever data with us. We equally appreciate the Disease Prevention and Control (DPC) Unit, World Health Organization Country Office, Abuja, Nigeria for providing resource materials on prevention of Lassa fever. 


\section{References}

[1]. Fichet-Calvet E, Rogers DJ. Risk maps of Lassa fever in West Africa. PLoS Negl Trop Dis. 2009; 3:388.

[2]. Bowen MD, Rollin PE, Ksiazek TG, Hustad HL, Bausch DG, Demby AH, Genetic diversity among Lassa virus strains. J Virol. 2000; 74:6992-7004.

[3]. Omilabu SA, Badaru SO, Okokhere P, Asogun D, Drosten C, Emmerich P, Lassa fever, Nigeria, 2003 and 2004. Emerg Infect Dis. 2005; $11: 1642-4$

[4]. Richmond JK, Banglole DJ. Lassa Fever: Epidemiology, Clinical Features, And Social Consequences". BMJ 2003; 327 (7426): 1271-1275.

[5]. Fisher-Hoch SP, Tomori O, Nasidi A, Perez-Oronoz GI, Fakile Y, Hutwagner L, McCormick JB. Review of cases of nosocomial Lassa fever in Nigeria: the high price of poor medical practice. BMJ 1995; 311: 857-859

[6]. Frame JD, Baldwin JM, Gocke DJ, Troup JM. Lassa fever, a new virus disease of man from West Africa: Clinical description and pathological findings. Am. J. Trop. Med. Hyg. 1970; 19: 670-6.

[7]. World Health Organization. WHO Lassa fever fact sheet No 179. Geneva:WHO, 2000

[8]. World Health Organization. "Lassa fever". Health Topics A to Z.

[9]. Tomori O, Fabiyi A, Sorungbe A, Smith A, McCormick JB. Viral hemorrhagic fever antibodies in Nigerian populations. Am J Trop Med Hyg 1988;38: 407-10.

[10]. McCormick JB, Webb PA, Krebs JW, Johnson KM, Smith ES. A prospective study of the epidemiology and ecology of Lassa fever. J Infect Dis 1987;155: 437-44. [PubMed]

[11]. Lukashevich LS, Clegg JC, Sidibe K. Lassa virus activity in Guinea: distribution of human antiviral antibody defined using enzyme-linked immunosorbent assay with recombinant antigen. J Med Virol 1993;40: 210-7. [PubMed]

[12]. Ogbu O, Ajuluchukwu E, Uneke CJ. "Lassa fever in West African sub-region: an overview". Journal of vector borne diseases 2007;44 (1): 1-11. 